\title{
Right heart dilatation in a fetus with an abnormal foramen ovale valve: an indicator of interatrial communication restriction
}

\author{
Wen-Jia Lei', Miao Fan², Mei-Lian Wang,4, Yu Wang1, Wei Sun', Xue Sun', Ying Zhang ${ }^{1}$ \\ ${ }^{1}$ Department of Sonography, Shengjing Hospital of China Medical University, Heping District, Shenyang, China, \\ ${ }^{2}$ Department of Radiology, The first Affiliated Hospital of Sun Yat-sen University, Guangzhou, China, ${ }^{3}$ Department of \\ Entomology, The Pennsylvania State University, University Park, PA 16802, USA, ${ }^{4}$ Department of Microbiology and \\ Parasitology, College of Basic Medical Sciences, China Medical University, Heping District, Shenyang, China
}

\begin{abstract}
Aims: Foramen ovale (FO) valve with a shape or motion abnormality is frequently detected during routine obstetric ultrasonic examinations. However, the hemodynamics mechanism of this entity remains unclear. The purpose of the study is to determine the relevance of interatrial communication restriction and resultant morphological modifications. Materials and methods: We reviewed the echocardiographic records of fetuses with isolated abnormal FO valve evaluated between January of 2010 and December of 2016. The size $\left(\mathrm{D}_{\mathrm{FO}}\right)$ of the FO orifice, opening angle $(\alpha)$ of the FO valve, and dimensions of cardiac chambers, FO channel outlet $\left(\mathrm{D}_{\mathrm{OUT}}\right)$ and inferior vena cava $\left(\mathrm{D}_{\mathrm{IVC}}\right)$ were measured. We evaluated their $\left(\mathrm{D}_{\mathrm{FO}}, \mathrm{D}_{\mathrm{OUT}}, \alpha\right)$ relationships to the diameters of RA and $\mathrm{D}_{\mathrm{IVC}}$. Five hundred and seventy normal fetuses were selected to establish the normal range of the $\mathrm{D}_{\mathrm{OUT}} / \mathrm{D}_{\mathrm{IVC}}$ ratio so as to provide a criterion for restriction. Results: An abnormal FO valve was identified in 89 fetuses without congenital heart disease, with restriction noted in 62 fetuses (45 fetuses with RA dilatation, 12 fetuses with RA and RV dilatation, and 5 fetuses with no RA dilatation). There were no significant correlations between RA/LA and $\mathrm{D}_{\mathrm{FO}} / \mathrm{D}_{\text {IVC }}, \mathrm{RA} /$ LA and $\alpha$. RA/LA was negatively correlated with $\mathrm{D}_{\mathrm{OUT}} / \mathrm{D}_{\mathrm{IVC}}\left(\mathrm{R}^{2}=0.97, \mathrm{p}<0.01\right)$. Conclusions: For a fetus with an abnormal FO valve, right heart dilatation could be considered as an indicator of interatrial communication restriction, which could be assessed by evaluating the FO channel outlet. The degree of right atrium dilatation indicates the severity of the restriction.

Keywords: fetal echocardiography; right atrial dilatation; restriction; foramen ovale valve; hemodynamics
\end{abstract}

\section{Introduction}

The atrial septum is an important intra-cardiac structure formed by the septum secundum of the right atrial side and the septum primum of the left atrial side [1]. The foramen ovale (FO) is formed on the septum secundum and must remain open during intrauterine life to maintain normal fetal circulation. The entrance of FO into the left atrium (LA) is covered by the FO valve, which is a ves-

Received 29.07.2017 Accepted 2.11.2017

Med Ultrason

2018, Vol. 20, No 2, 213-220

Corresponding author: Ying Zhang

Department of Sonography, Shengjing Hospital of China Medical University 36 Sanhao Street, Heping District, Shenyang 110004, Liaoning Province, China.

Phone: 18940258676, Fax: 0086-024-85838633

E-mail: baogoubei@hotmail.com tige of the septum primum [2,3]. Normally, the FO valve appears freely mobile and nonrestrictive, with a smooth curvature bulging into the LA [4]. When the FO valve adheres to the septum secundum with reduced or absent movement or when two FO valves stick end-to-end, which appears like an atrial septal aneurysm, an interatrial communication restriction might be present $[4,5]$.

Mechanisms of interatrial communication restriction include a narrow FO orifice and FO valve with a shape or motion abnormality. The latter pathology may not be detected by measuring only the diameter of the FO orifice, as is often suggested in the literature [6-9]. Once the abnormal FO valve is identified, the blood that enters the FO orifice still has to travel through a channel formed by the FO valve and the septum secundum (in the case of a fixed FO valve) or a channel formed by the two FO valves (in the case of an atrial septal aneurysm). Based on this concept, a study of postmortem specimens by Pat- 
ten et al showed that the outlet portion of FO channel represents the restricting structure during normal intrauterine development [10].

In the current study, we aimed to determine if an abnormal FO valve has relevance to the interatrial communication restriction. We also analyzed the hemodynamics of those fetuses with abnormal FO valve for evaluating the factors responsible for the severity of interatrial communication restriction.

\section{Materials and methods}

\section{Study Population}

We retrospectively reviewed the imaging data obtained from the fetal echocardiography center in our hospital from January of 2010 to December of 2016. The subjects comprised a normal group and a case group. During the study period, the echocardiographic records of normal fetuses diagnosed in our hospital were reviewed. Those without high-quality images of the sagittal views for measuring the diameter of the FO channel outlet were excluded. Fetuses of the gravida with smoking, diabetes, hypertension, or any general chronic disease were also excluded. All fetuses were singletons. The case group was selected from cases in which the FO valve had a shape or motion abnormality. The FO valve was diagnosed as a shape abnormality when it appeared to be either aneurysmal or hypermobile as previously described [11-13]; or motion abnormality, when it appeared to be thick and flat without a "flapping" motion normally seen in the uterus $[14,15]$. The cases diagnosed with congenital heart diseases or with no clear images were excluded. Outcome data was obtained from postnatal echocardiograms.

All fetuses were examined using the Voluson 730, E8, and E10, GE Healthcare, Kretztechnik, Zipf, Austria ultrasound systems. Routine obstetric sonography was initially performed to detect extra-cardiac malformations. Then, a detailed cardiac examination was performed by an experienced fetal echocardiographer (Z.Y.). The visceral and cardiac positional relationship was determined as previously described [16]. Four transverse views including the four-chamber view (4CV), the left and right outflow tract views, and the three-vessel-trachea view were scanned. In addition, three sagittal views including the bicaval view, the aortic arch view, and the ductal arch view were also obtained. High-definition flow imaging (HDFI) was performed when necessary. All imaging data was saved as video clips for later analysis.

Study design and fetal cardiac image interpretation

For the normal group, the distance $\left(\mathrm{D}_{\mathrm{N}-\mathrm{OUT}}\right)$ between the final point of the FO valve and the atrial septum and the dimension $\left(\mathrm{D}_{\mathrm{IVC}}\right)$ of the inferior vena cava (IVC) were meas- ured in the sagittal views. Data of $\mathrm{D}_{\mathrm{N}-\mathrm{OUT}} / \mathrm{D}_{\mathrm{IVC}}$ was plotted using a linear regression with $95 \%$ distribution range for individual observations. Diagnosis of interatrial communication restriction was made on the basis of the criterion that the ratio is less than the lower limit of $95 \%$ reference range.

According to our criterion, the case group was separated into two groups including a restriction group and a non-restriction group. Figure 1a shows the measurement of the diameters of cardiac chambers. As established by Tan J, et al [6], right atrium (RA) dilatation between 26 and 32 gestational weeks (GW) was defined as an estimated RA/LA $>1.1$ and defined as $>1.2$ at $32 \mathrm{GW}$ and above. Right ventricle (RV) dilatation was defined as an estimated RV/LV $>1.3$ (normal ratio=1.18) [17]. Fetuses in the restriction group were assigned to three subgroups according to alterations of the cardiac chambers: isolated RA dilatation (Group A), RV and RA dilatation (Group $\mathrm{B}$ ), and no RA or RV dilatation (Group C). The size $\left(\mathrm{D}_{\mathrm{FO}}\right)$ of the FO orifice was measured in the 4CV. Besides, we also measured an angle $(\alpha)$ in the sagittal views, which could represent the shape or extent of the distension of FO valve in some degree. A sketch map shows this method of measuring $\alpha$ (fig $1 \mathrm{~b}$ ). The maximal value of the FO channel outlet $\left(\mathrm{D}_{\text {OUT }}\right)$ and $\mathrm{D}_{\text {IVC }}$ were obtained in the sagittal views. The relationships of RA/LA (and RV/LV)

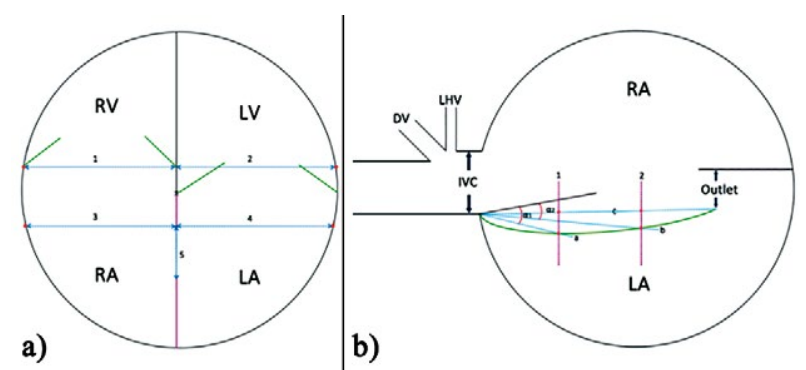

Fig 1. a) sketch map measuring the dimensions of cardiac structures. Measuring the diameters of the RV (indicated as 1) and the LV (indicated as 2) in the end diastolic phase just below the level of the atrioventricular valves; measuring the diameters of the RA (indicated as 3) and the LA (indicated as 4) in the end systolic phase at the level of the edge of the secundum septum; and measuring the size of FO orifice (indicated as 5) in the $4 \mathrm{CV}$. FO, foramen ovale; LA, left atrium; LV, left ventricle; RA, right atrium; RV, right ventricle; b) sketch map measuring the $\alpha\left({ }^{\circ}\right.$ ). First, draw a straight line (line c) between the two ends of the FO valve. Then, divide line $\mathrm{c}$ into three equal parts and draw two lines that are perpendicular to line $\mathrm{c}$ (line 1 and line 2 ). There are two intersections of line 1 and line 2 with the FO valve (indicated as a green line). Finally, draw two lines (line a and line b) from two intersections through the adhering point of the FO valve to the margin of the secundum septum, forming two angles between line a and the secundum septum $(\alpha 1)$, as well as between line $b$ and the secundum $(\alpha 2)$. The average value $(\alpha)$ of $\alpha 1$ and $\alpha 2$ can be calculated. DV, ductus venosus; LA, left atrium; LHV, left hepatic vein; IVC, inferior vena cava; RA, right atrium. 
to $\mathrm{D}_{\mathrm{FO}} / \mathrm{D}_{\mathrm{IVC}}, \mathrm{RA} / \mathrm{LA}$ (and RV/LV) to $\alpha$, and RA/LA (and $\mathrm{RV} / \mathrm{LV}$ ) to $\mathrm{D}_{\mathrm{OUT}} / \mathrm{D}_{\mathrm{IVC}}$ were analyzed. HDFI technique was used to detect the full course of the IVC draining into the LA through the outlet.

In addition, the mean $\alpha$, the mean $\mathrm{D}_{\mathrm{FO}} / \mathrm{D}_{\mathrm{IVC}}$ ratio and the mean $\mathrm{D}_{\mathrm{OUT}} / \mathrm{D}_{\mathrm{IVC}}$ ratio were calculated and compared among groups (Groups A, B, C, and non-restriction group). Data of $\mathrm{D}_{\mathrm{OUT}} / \mathrm{D}_{\mathrm{IVC}}$ for fetuses with RA dilatation (Groups A and Group B) was also represented graphically. The data was plotted against GW and compared with that of normal group.

\section{Statistical Analysis}

Data was expressed as mean \pm standard deviation (SD). Data was analyzed using Prism (GraphPad, La Jolla, CA). A simple linear regression analysis was used to evaluate relationships between the variables of interest. Student's $t$-test was used to compare mean values. The chi-squared test was used to assess statistical relationships between categorical data. Statistical relationships were considered significant at $\mathrm{p}<0.05$.

\section{Results}

\section{Abnormal FO valve characteristics}

There were two predominant types of abnormal FO valve demonstrated in our study. A type of motion abnormality, which was thick and did not have the typical swinging motion during the cardiac cycle, was documented in 23 (25.8\%) fetuses. Two video clips illustrate this in greater detail (video 1 and video 2, on the journal site). The FO channel outlet was the orifice formed by the single fixed FO valve and the septum secundum, which could be observed in the $4 \mathrm{CV}$ and the sagittal views (fig $2 a, b)$. Fig $2 c$ shows only a small amount of flow entering the LA through a narrow outlet in a sagittal view by HDFI. An additional video clip illustrates this in greater detail (video 3 , on the journal site). The structure of the outlet is best illustrated by the sketch map (fig $2 \mathrm{~d}$ ). The remaining $66(74.2 \%)$ fetuses demonstrated the typical presentations of atrial septal aneurysm, which was formed by two hypermobile and thin valves and appeared to balloon into the LA (fig 3a,b). An additional video clip illustrates this in greater detail (video 4, on the journal site). The FO channel outlet was formed by the final portions of two FO valves, which adhered to the septum secundum. An additional video clip illustrates this in greater detail (video 5, on the journal site). A sketch map illustrates the structure of this type of outlet (fig 3c).

\section{Distribution of all groups and outcome of statistical analysis}

In figure 4 the subjects, distribution of all groups, and statistical analysis described in the study design are

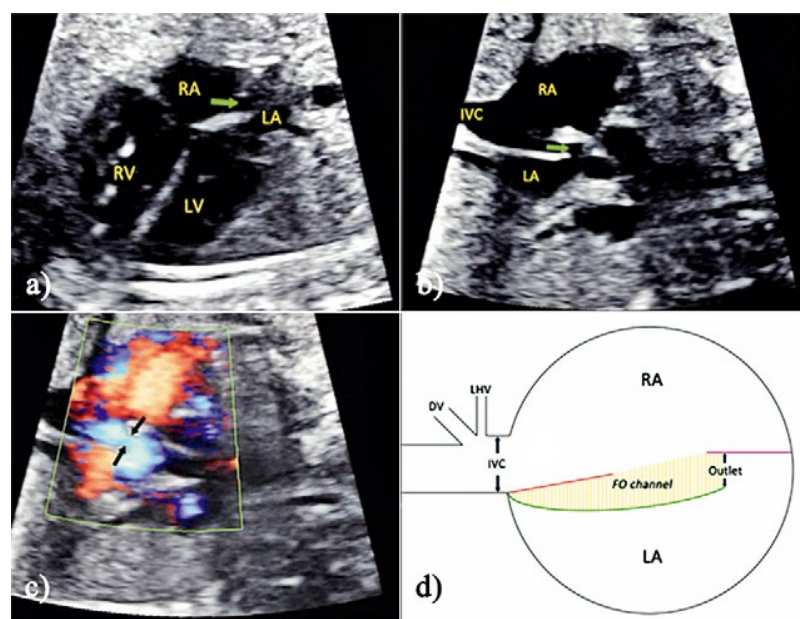

Fig 2. Images of a fetus with a fixed FO valve at 33 weeks gestation. 4CV showed a small orifice (indicated by an arrow) formed by a single fixed $\mathrm{FO}$ valve and the septum secundum (a). A sagittal view clearly shows the narrow outlet (indicated by an arrow) formed by a single fixed FO valve and the septum secundum (b). By HDFI, only a small amount of flow (indicated by arrows) can be identified entering the LA through the narrow outlet (c). A sketch map of a sagittal view illustrating how the FO channel outlet is formed by a fixed FO valve (indicated as a green line) and the septum secundum (d). DV, ductus venosus; FO, foramen ovale; HDFI, high-definition flow imaging; IVC, inferior vena cava; LA, left atrium; LV, left ventricle; LHV, left hepatic vein; RV, right ventricle; RA, right atrium.

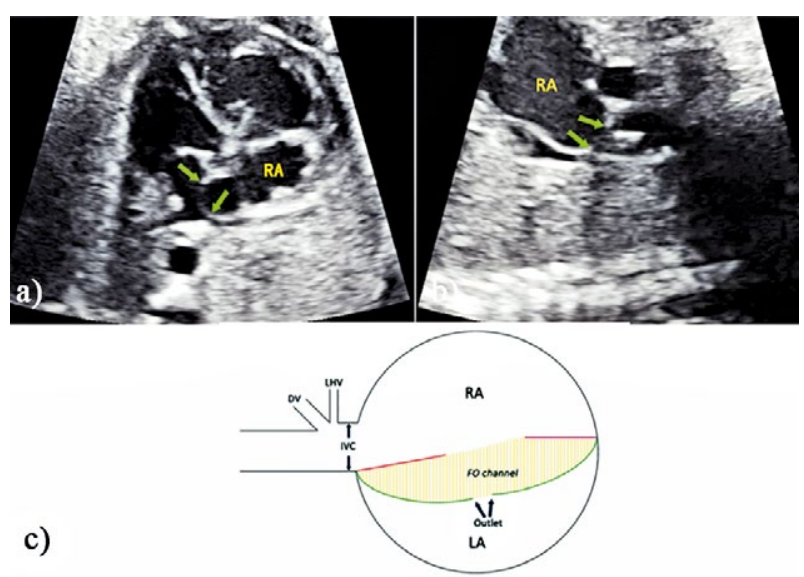

Fig 3. Images of a fetus with an atrial septal aneurysm at 30 weeks gestation. 4CV shows an indistinct gap formed by the ends of two FO valves (indicated by arrows) (a). A sagittal view shows the outlet clearly formed by the ends of two FO valves (indicated by arrows) (b). A sketch map of the sagittal view illustrating how the FO channel outlet is formed by the ends of two FO valves (indicated by arrows) (c). DV, ductus venosus; FO, foramen ovale; IVC, inferior vena cava; LA, left atrium; LHV, left hepatic vein; RA, right atrium. 
shown. During the study period, 1637 fetuses underwent cardiac evaluation. No structural heart defect was found in 960 fetuses and 570 fetuses were selected for the normal group. Age in GW ranged from 21 to 39 (median, 30.0). Maternal age ranged from 20 to 33 years old (median, 28.0). Abnormal FO valves were found in 119 fetuses resulting in a prevalence of $12.4 \%$ among this highly selected cohort; 89 fetuses were selected into case group.

In Table I are detailed the number the fetuses selected in the five groups and the data of $\alpha, \mathrm{D}_{\mathrm{FO}} / \mathrm{D}_{\mathrm{IVC}}$, and

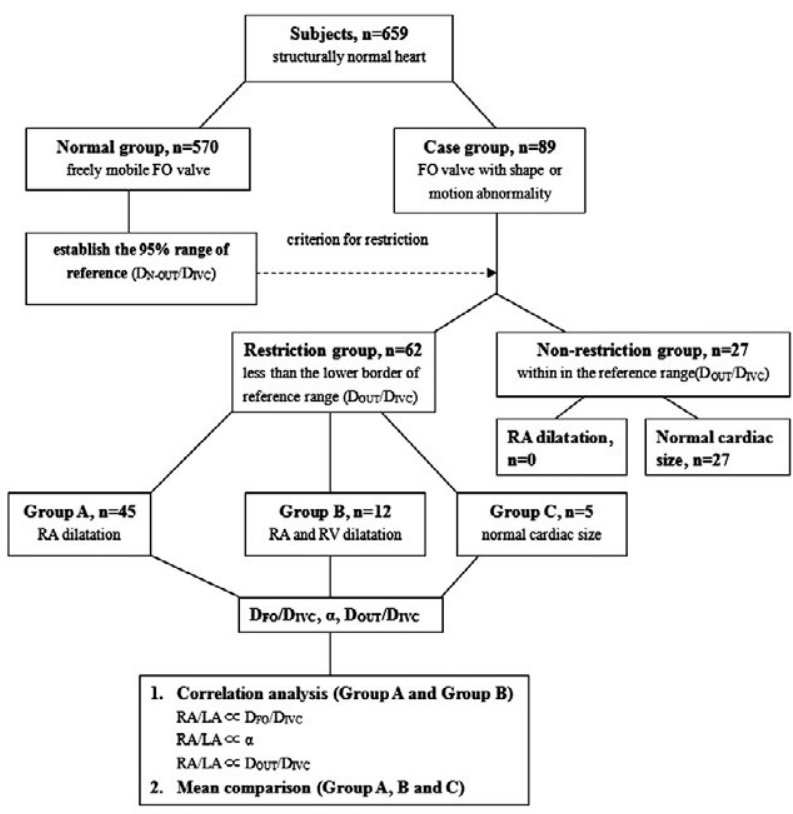

Fig 4. Subjects, group distribution and statistical analysis in our study. $\alpha$, an angle indicating the degree of distention of the FO valve; DFO, the size of the FO orifice; DIVC, the dimension of the inferior vena cava; DN-OUT, the distance between the final point of the $\mathrm{FO}$ valve and the atrial septum in normal group; DOUT, the maximal value of the FO channel outlet in case group; FO, foramen ovale; LA, left atrium; RA, right atrium.
$\mathrm{D}_{\text {OUT(N-OUT) }} / \mathrm{D}_{\text {IVC }}$ obtained in these groups. There were no remarkable differences in mean $\alpha$ and mean $\mathrm{D}_{\mathrm{FO}} / \mathrm{D}_{\mathrm{IVC}}$ ratio among Groups $\mathrm{A}, \mathrm{B}, \mathrm{C}$ and non-restriction group. However, the mean $\mathrm{D}_{\mathrm{OUT}} / \mathrm{D}_{\mathrm{IVC}}$ ratio in Group $\mathrm{B}$ was lower than that observed in Group A and Group $C(p<0.01$, respectively). Also, the mean $\mathrm{D}_{\mathrm{OUT}} / \mathrm{D}_{\mathrm{IVC}}$ ratio in Groups $\mathrm{A}, \mathrm{B}, \mathrm{C}$ was lower than that of non-restriction group $(\mathrm{p}<0.01$, respectively $)$.

For fetuses with RA dilatation (Groups A and Group $B)$, there were no significant correlations between RA/ $\mathrm{LA}$ and $\mathrm{D}_{\mathrm{FO}} / \mathrm{D}_{\text {IVC }}\left(\mathrm{R}^{2}=0.03, \mathrm{p}=0.18\right)$ or $\mathrm{RA} / \mathrm{LA}$ and $\alpha$ $\left(\mathrm{R}^{2}=0.01, \mathrm{p}=0.57\right)$. However, $\mathrm{RA} / \mathrm{LA}$ was negatively correlated with $\mathrm{D}_{\text {OUT }} / \mathrm{D}_{\text {IVC }}\left(\mathrm{R}^{2}=0.97, \mathrm{p}<0.01\right)$. Notably, for fetuses with RV dilatation (Group B), the RV/LV ratio had no significant correlation with $\mathrm{D}_{\mathrm{FO}} / \mathrm{D}_{\mathrm{IVC}}\left(\mathrm{R}^{2}=0.003\right)$, $\alpha\left(\mathrm{R}^{2}=0.02\right)$, or $\mathrm{D}_{\text {OUT }} / \mathrm{D}_{\text {IVC }}\left(\mathrm{R}^{2}<0.01\right)$. This analysis was limited by the number of samples.

Linear regressions between $\mathrm{D}_{\text {OUT }} / \mathrm{D}_{\text {IVC }}\left(\mathrm{D}_{\mathrm{N}-\mathrm{OUT}} / \mathrm{D}_{\mathrm{IVC}}\right)$ and $\mathrm{GW}$ were performed. The lower limit of $95 \%$ reference range remained at 0.5-0.6 during second pregnancy. All cases with RA dilatation (Group A and Group B) were under the lower limit of $95 \%$ reference range.

\section{Follow-up and prognosis}

For fetuses in the case group, fetal echocardiogram was performed on a regular basis at least every week for potential existence of restriction and later evolutive signs. For the non-restriction group, GW at first diagnosis ranged from 26 to 39 weeks (median, 32.7). There was no RA dilatation or other signs of restriction during the follow-up period. For the restriction group, GW at first diagnosis ranged from 26 to 36 weeks (median, 30.4). Five cases that were lost to follow-up were excluded. There were 40 fetuses with stable hemodynamic state and 17 cases with evolutive signs of restriction (Table II).

In our study, the prognosis of all fetuses with abnormal FO valve was good. Fifty-two of 57 fetuses with RA dilatation showed a normal RA within the first 2 weeks of life, as determined by the postnatal echocardiogram.

Table I. Detailed data of $\alpha$, DFO/DIVC, and DOUT(N-OUT)/DIVC among the five groups of the study.

\begin{tabular}{llllll}
\hline Group & & $\mathbf{n}$ & $\boldsymbol{\alpha}\left(^{\circ}\right)$ & DFO/DIVC & DOUT(N-OUT)/DIVC \\
\hline Restriction group & Group A & 45 & $56.56 \pm 16.40$ & $1.12 \pm 0.15$ & $0.45 \pm 0.08^{* \dagger}$ \\
& Group B & 12 & $59.92 \pm 16.28$ & $1.01 \pm 0.14$ & $0.32 \pm 0.14^{* \dagger}$ \\
& Group C & 5 & $52.40 \pm 3.85$ & $1.11 \pm 0.15$ & $0.51 \pm 0.06^{* \dagger}$ \\
Non-restriction group & & 27 & $55.48 \pm 9.21$ & $1.02 \pm 0.11$ & $1.02 \pm 0.19^{\dagger}$ \\
Normal group & & 570 & NA & NA & $0.95 \pm 0.24$ \\
p value & NA & NA & NS & NS & $\mathrm{S}^{* \dagger}$ \\
\hline
\end{tabular}

Group A, fetuses with isolated RA dilatation; Group B, fetuses with RA and RV dilatation; Group C, fetuses with no RA dilatation; $\alpha$, an angle indicating the opening range of the FO valve; DOUT, the maximal value of the FO channel outlet; DN-OUT, the distance between the final point of the FO valve and the atrial septum in normal group; DIVC, the dimension of the inferior vena cava; DFO, the size of the FO orifice; FO, foramen ovale; RA, right atrium; NA, not applicable; NS, not significant; S, significant. Data presented as mean \pm SD. ${ }^{*}$ Group B compared with Group A and Group C, p $<0.01$. †Non-restriction group compared with Group A, B and C, p<0.01. 
Table II. Follow-up results of fetuses with evolutive signs of restriction.

\begin{tabular}{lllllll}
\hline Case & $\begin{array}{l}\text { GW at diagnosis } \\
\text { (weeks) }\end{array}$ & $\begin{array}{l}\text { Evolutive signs of } \\
\text { restriction }\end{array}$ & $\begin{array}{l}\text { GW at progression } \\
\text { (weeks) }\end{array}$ & $\begin{array}{l}\text { Flow through FO } \\
\text { channel }\end{array}$ & $\begin{array}{l}\text { GW at birth } \\
\text { (weeks) }\end{array}$ & $\begin{array}{l}\text { Mode of } \\
\text { delivery }\end{array}$ \\
\hline $1^{*}$ & 26 & EDRA & 29 & constant & 37 & S \\
$2^{*}$ & 26 & EDRA & 33 & constant & 36 & S \\
$3^{*}$ & 27 & EDRA & 35 & decreased & 38 & S \\
$4^{*}$ & 28 & EDRA & 36 & constant & 38 & CS \\
$5^{*}$ & 28 & EDRA & 37 & constant & 38 & CS \\
6 & 30 & EVD & 34 & decreased & 37 & S \\
7 & 30 & EVD & 36 & constant & 38 & CS \\
8 & 30 & PTR & 36 & decreased & 37 & S \\
9 & 30 & PTR & 34 & decreased & 35 & 38 \\
10 & 32 & EVD & 33 & decreased & 38 & CS \\
11 & 32 & EVD & 35 & constant & 37 & CS \\
12 & 32 & PTR & 36 & decreased & nearly disappeared & 35 \\
13 & 32 & PE & 35 & decreased & 38 & S \\
14 & 35 & PDRA & 37 & decreased & 37 & CS \\
15 & 35 & PDRA & 37 & decreased & 37 & S \\
16 & 35 & PTR & PDRA & 37 & decreased & 37 \\
\hline
\end{tabular}

CS, caesarean section; EDRA: emerging dilatation of right atrium; EVD, emerging ventricular disproportion; GW, gestational week; PDRA, progressive dilatation of right atrium; PE, pericardial effusion; PTR, progressive tricuspid regurgitation; S, spontaneous. *Fetuses with normal cardiac chambers at the time of diagnosis of interatrial communication restriction.

Table III. Prenatal morphologic findings and postnatal outcomes of all fetuses in our case series

\begin{tabular}{|c|c|c|c|c|c|c|c|c|c|}
\hline \multirow[t]{2}{*}{ Fetuses } & & \multicolumn{3}{|c|}{ Prenatal morphologic findings (n) } & \multicolumn{5}{|c|}{ Postnatal abnormalities (n) } \\
\hline & & Normal & $\begin{array}{l}\text { Isolated RA } \\
\text { dilatation }\end{array}$ & $\begin{array}{l}\text { RA and RV } \\
\text { dilatation }\end{array}$ & RA dilatation & FO & ASD & PDA & TR* \\
\hline Normal group & & 570 & NA & NA & None & 147 & 47 & 19 & None \\
\hline \multirow[t]{2}{*}{ Case group } & Aneurysmal & 26 & 31 & 9 & 4 & 21 & 5 & 3 & 4 \\
\hline & Fixed & 6 & 14 & 3 & 1 & 9 & 3 & None & 1 \\
\hline
\end{tabular}

*Moderate or severe tricuspid regurgitation (TR) (defined as peak velocities above $3.0 \mathrm{~m} / \mathrm{s}$ ). ASD, atrial septal defect; FO, foramen ovale; NA, not applicable; PDA, patent ductus arteriosus; RA, right atrium; RV, right ventricle.

Another 5 neonates showed an enlarged RA with varying degrees of TR. Table III show these results in detail.

\section{Discussions}

FO valve with shape or motion abnormality is frequently detected during routine obstetric ultrasonic examinations as it is easy to be visualized in the $4 \mathrm{CV}$. However, there remains some confusion whether abnormal FO valve belongs to normal variations or may affect fetal cardiac hemodynamic. The results of the current study indicated that interatrial communication restriction may exist in parts of these fetuses with resultant right heart dilatation. We also demonstrated that a restriction is not present when the cardiac chamber is within the normal range during the late pregnancies. Accurate evaluation of the FO channel outlet is the key factor to decide whether a restriction exists when abnormal $\mathrm{FO}$ valve are present.
In fact, restrictive FO has long been noticed by fetal echocardiographers. Previous reports mainly focused on the dimension of the FO orifice [7-9]. However, these diagnosis standards of restrictive FO are obviously not satisfactory as they could not show the full hemodynamics of interatrial communication. The measurement of blood velocity across FO was a development. However, accuracy might be affected as Doppler shift is angle-dependent. Recently, Patten et al [10] proposed the conception of the FO channel and indicated that the outlet portion of this channel could make the assessment of the interatrial communication function more complete. On this basis, Kiserud et al [18] established the normal range of the area ratio between the FO channel outlet and IVC. In our study, we measured the diameter of the FO channel outlet. A relatively large number of normal fetuses were included to establish the reference range. The results showed a high consistency with that of Kiserud et al. As 
the diameter of both the FO channel outlet and IVC could be easily measured when compared with measuring the area, the obstetric sonographers and fetal echocardiographers are apt to use our newly proposed standard for evaluating fetal interatrial communication.

As is well known, the physiologic interatrial shunt enables the oxygenated blood entering the right heart to be ejected to the left heart, which is important to ensure a stable hemodynamics state between the left heart and the right heart [19]. Intrauterine restriction of interatrial communication might cause the increased blood flow into the right heart and decreased flow into the left heart with resultant morphologic abnormalities, such as right heart dilatation [20]. Theoretically, the chambers may not dilate when only mild restriction is present. However, RA will dilate, and then with the presence of the enlargement of $\mathrm{RV}$, when the restriction is moderate or severe. The results of the current study confirmed the hypothesis: $\mathrm{D}_{\text {OUT }}$ $\mathrm{D}_{\mathrm{IVC}}$ was negatively correlated with RA/LA. In addition, fetuses with both RA and RV dilatation exhibited a lower $\mathrm{D}_{\text {OUT }} / \mathrm{D}_{\text {IVC }}$ ratio than those with isolated RA dilatation. We believe that $\mathrm{D}_{\text {OUT }} / \mathrm{D}_{\text {IVC }}$ could be used to quantitatively analyze the severity of restriction.

According to our results, not all fetuses with abnormal FO valve presented the restriction. We measured an angle $(\alpha)$, which could represent the shape of the FO valve to some extent. It showed no correlation with RA/ LA, indicating that the isolated abnormal shape of the FO valve could not lead to the restriction. The results could explain that not all fetuses with atrial septum aneurysm would present the restriction. The restriction could only be diagnosed when the FO channel outlet was accurately evaluated. We believe that the sagittal views are best suited for evaluating the FO channel outlet, particularly since it is based on the actual flow direction in the atrial area as evidenced by prior studies $[18,21,22]$. Besides, according to our experience, it is impossible to identify the FO channel outlet in the $4 \mathrm{CV}$ in most cases.

However, it is a challenge for an obstetric sonographer to scan qualified sagittal views and to evaluate the FO channel during routine examinations. Instead, the alterations of cardiac chambers could be easily identified. According to our results, the majority fetuses with abnormal $\mathrm{FO}$ valve and interatrial communication restriction showed dilated RA, or combined with RV dilatation. On the contrary, fetuses with abnormal FO valve while with no restriction showed no alterations of cardiac chambers. This suggests that RA/LA could be used to predict the existence of restriction, when abnormal FO valve presents.

It was worth noting that the RA volume overload, RA pressure overload, or ventricular disproportion may develop as a progression of earlier restriction. In the cur- rent study, 5 fetuses enrolled in the second trimester evidenced symmetrical left and right chambers. However, RA dilatation appeared in late pregnancy. We speculate that the normal cardiac dimension at the initial diagnosis resembles the compensatory ability of the right heart during the early stage of restriction.

In fact, a close follow-up is necessary when evidence shows interatrial communication restriction, whether RA dilatation exists or not. The examiner should be concerned with the appearance of RA dilatation, or progressive right heart dilatation with fetal hydrops, and tricuspid valve insufficiency $[4,5,12]$. In addition, the prognosis of interatrial communication restriction also depends at the point of time when RA dilatation appears and the severity of restriction [4]. The earlier and the more severe RA dilatation appears, the easier progressive restriction may present. However, obstetricians should not be too pessimistic about the prognosis of interatrial communication restriction. Hagen et al. reported that, as long as the fetus remained hemodynamically stable, the prognosis of an interatrial communication restriction was good and the pregnancy should be allowed to continue until term with close monitoring [1]. In the current study, all fetuses with restriction had a good result. Various degrees of RA dilatation, or combined with RV enlargement presented, resembled right heart volume overload. During the follow-up period, most cases maintained a stable hemodynamic state. Signs of progressive right heart volume overload presented in only 17 fetuses, 7 of which delivered at full term with close monitoring, while the rest delivered prematurely with adequate gestational age and lung maturity. For intrauterine interatrial communication restriction, obstetricians and sonographers should identify the abnormality as early as possible. We believe that a close monitoring ensures proper strategies for the management of these fetuses, and in turn, guarantees a good prognosis.

The main limitation of this study is that it was a mono-center respective review. Furthermore, fetuses without high-quality sagittal views were excluded from the study which may cause potential selective bias.

\section{Conclusions}

We proposed an easy method with reference range herein to identify intrauterine restriction of interatrial communication. For fetuses with abnormal shape or motion of FO valve, the dilatation of the right heart could be considered as an indicator of interatrial communication restriction. The larger RA/LA ratio indicates a more severe restriction. Evaluation of the FO channel outlet in the sagittal views guarantees an accurate assessment of the restriction. 


\section{Acknowledgements}

The authors gratefully acknowledge the support of Professor Wei-Dong Ren, Chair of the Department of Sonography, Shengjing Hospital of China Medical University, Liaoning Province, China, for his great assistance in this study.

\section{Conflict of interest: none}

\section{References}

1. Uzun O, Babaoglu K, Ayhan YI, et al. Diagnostic ultrasound features and outcome of restrictive foramen ovale in fetuses with structurally normal hearts. Pediatr Cardiol 2014;35:943-952.

2. Sweeney LJ, Rosenquist GC. The normal anatomy of the atrial septum in the human heart. Am Heart J 1979;98:194199.

3. Campbell M. Natural history of atrial septal defect. $\mathrm{Br}$ Heart J 1970;32:820-826.

4. Chobot V, Hornberger LK, Hagen-Ansert S, Sahn DJ. Prenatal detection of restrictive foramen ovale. J Am Soc Echocardiogr 1990;3:15-19.

5. Hagen A, Albig M, Schmitz L, et al. Prenatal diagnosis of isolated foramen ovale obstruction. A report of two cases. Fetal Diagn Ther 2005;20:70-73.

6. Tan J, Silverman NH, Hoffman JI, Villegas M, Schmidt KG. Cardiac dimensions determined by cross-sectional echocardiography in the normal human fetus from 18 weeks to term. Am J Cardiol 1992;70:1459-1467.

7. Phillipos EZ, Robertson MA, Still KD. The echocardiographic assessment of the human fetal foramen ovale. J Am Soc Echo 1994; 7:257-263.

8. Feit LR, Copel JA, Kleinman CS. Foramen ovale size in the normal and abnormal human fetal heart: an indicator of transatrial flow physiology. Ultrasound Obstet Gynecol 1991;1:313-319.

9. Wilson AD, Rao PS, Aeschlimann S. Normal fetal foramen ovale flap and transatrial Doppler velocity pattern. J Am Soc Echocardiogr 1990;3:491-494.

10. Patten BM, Sommerfield WA, Paff GH. Functional limitations of the foramen ovale in the human foetal heart. Anat Rec 1929;44:165-178.

11. Tuo G, Paladini D, Montobbio G, Volpe P, Cheli M, Calevo MG, Marasini M. Prenatal echocardiographic assessment of foramen ovale appearance in fetuses with d-transposition of the great arteries and impact on neonatal outcome. Fetal Diagn Ther 2017;42:48-56.

12. Sun HY, Fripp RR, Printz BF. Unusual consequence of a fetal atrial septal aneurys. Clin Case Rep 2015;3:368-369.

13. Channing A, Szwast A, Natarajan S, Degenhardt K, Tian Z, Rychik J. Maternal hyperoxygenation improves left heart filling in fetuses with atrial septal aneurysm causing impediment to left ventricular inflow. Ultrasound Obstet Gynecol 2015;45:664-669.
14. Amaral HB, Zielinsky P, da Silveira AF, et al. Morphological basis for the study of the interatrial septum in the human fetus. Arq Bras Cardiol 2007;88:559-564.

15. Maeno YV, Kamenir SA, Sinclair B, van der Velde ME, Smallhorn JF, Hornberger LK. Prenatal features of ductus arteriosus constriction and restrictive foramen ovale ind-transposition of the great arteries. Circulation 1999;99:1209-1214.

16. Zhang Y, Fan M, Ren WD, et al. Prenatal diagnosis of fetal unilateral lung agenesis complicated with cardiac malposition. BMC Pregnancy Childbirth 2013;13:79.

17. Sahn DJ, Lange LW, Allan HD, et al. Quantitative real-time cross-sectional echocardiography in the developing normal human fetus and newborn. Circulation 1980;62:588-597.

18. Kiserud T, Rasmussen S. Ultrasound assessment of the fetal foramen ovale. Ultrasound Obstet Gynecol 2001;17:119124.

19. Talemal L, Donofrio MT. Hemodynamics consequences of a restrictive ductus arteriosus and foramen ovale in fetal transposition of the great arteries. J Neonatal Perinatal Med 2016;9:317-320.

20. Enzensberger C, Degenhardt J, Vogel M, et al. Fetal pulmonary venous flow and restrictive foramen ovale in hypoplastic left heart. Ultrasound Obstet Gynecol 2012;40:17.

21. Kiserud T, Eik-Nes SH, Blaas HG, Hellevik LR. Foramen ovale: an ultrasonographic study of its relation to the inferior vena cava, ductus venosus and hepatic veins. Ultrasound Obstet Gynecol 1992;2:389-396.

22. Lind J, Wegelius C. Angiocardiographic studies on the human foetal circulation; a preliminary report. Pediatrics 1949;4:391-400.

\section{Additional Files}

Video 1. 2D imaging showing the $4 \mathrm{CV}$ of a fetus with a single fixed FO valve at 33 weeks gestation. A narrow gap (indicated by an icon) formed by a single fixed FO valve and the septum secundum is visualized. FO, foramen ovale; LV, left ventricle; RA, right atrium; RV, right ventricle.

Video 2. 2D imaging showing a sagittal view of the same fetus as in Video 1. A clear outlet (indicated by an icon) formed by a single fixed FO valve and the septum secundum is visualized. The FO valve did not have the typical swinging motion. FO, foramen ovale; IVC, inferior vena cava; RA, right atrium.

Video 3. Simultaneous imaging of gray-scale and high-definition flow imaging showing a sagittal view of the same fetus as in Video Files 1 \& 2. By high-definition flow imaging, only a small amount of flow (indicated by an arrow) could be identified entering into the LA through the narrow outlet formed by a single fixed FO valve and the septum secundum. FO, foramen ovale; LA, left atrium; RA, right atrium.

Video 4. 2D imaging showing the $4 \mathrm{CV}$ of a fetus with an atrial septal aneurysm at 30 weeks gestation. Two FO valves 
with an indistinct gap (indicated by an arrow) at their final portions are visualized. AO, aorta; FO, foramen ovale; $\mathrm{LV}$, left ventricle; RA, right atrium; RV, right ventricle.
Video 5. 2D imaging showing a sagittal view of the same fetus as in Video File 4. A clear outlet (indicated by an icon) formed by the ends of two $\mathrm{FO}$ valves is visualized. $\mathrm{AO}$, aorta; FO, foramen ovale; IVC, inferior vena cava; RA, right atrium. 